\title{
Recent topics in graft-versus-host disease: from the perspectives of pathogenesis and treatment
}

\author{
Makoto Murata ${ }^{1}$
}

Received: 19 March 2015 / Revised: 24 March 2015 / Accepted: 25 March 2015 / Published online: 2 April 2015

(C) The Japanese Society of Hematology 2015

Sustained efforts to conquer graft-versus-host disease (GVHD), a life-threatening complication after allogeneic hematopoietic stem cell transplantation (HSCT), have been made over the years. However, the pathogenesis remains to be completely elucidated, and optimization of prophylaxis and therapy for this condition has thus not yet been accomplished. GVHD occurs in more than half of allogeneic HSCT recipients, and steroid-refractory GVHD carries a particularly poor prognosis. Nonetheless, recent research has provided opportunities to better understand the pathogenesis of GVHD and develop new strategies for prophylaxis and therapy of GVHD.

In the "Progress in Hematology" feature in this issue, three excellent review articles [1-3] and my own [4] cover recent topics in the pathogenesis and treatment of GVHD. The first article focuses on interactions between intestinal microbiota and the development of GVHD. The second focuses on the role of B-cell immunity in chronic GVHD. Both topics have received extensive attention in recent years, as advances in our understanding the pathogenesis of GVHD contribute directly to the development of more effective prophylaxis and therapy for GVHD. The third and last articles present the latest clinical developments in acute and chronic GVHD treatments in other countries and Japan, respectively.

Early clinical studies suggested a benefit of near-total bacterial decontamination in terms of reducing GVHD, as well as prophylaxis for infection. However, later studies

Makoto Murata

mmurata@med.nagoya-u.ac.jp

1 Department of Hematology and Oncology, Nagoya University Graduate School of Medicine, 65 Tsurumai, Showa, Nagoya, Aichi 466-8550, Japan showed no clear benefit and consensus is lacking regarding the ideal choice of prophylactic antibiotic coverage. A new technology, deep-sequencing of $16 \mathrm{~S}$ rRNA, allows characterization of the composition of mixed bacterial samples and re-examination of associations between individual intestinal microbial flora and GVHD development. Shono et al. [1] at the Memorial Sloan Kettering Cancer Center outline the biology of intestinal homeostasis in relation to the microbiota in the setting of allogeneic HSCT, and clearly delineate the interactions between intestinal microbiota and their impact on GVHD.

Chronic GVHD presents with diverse symptoms, such as sicca syndrome, skin sclerosis, and pulmonary involvement, and is associated with a high risk of long-term morbidity and mortality. The pathogenesis of chronic GVHD remains poorly understood, but the evidence increasingly supports the importance of B-cell immunity in the development of chronic GVHD. Nakasone et al. [2] at Stanford University School of Medicine describe how B cells contribute to chronic GVHD pathogenesis and discuss the efficacy of B-cell depletion and modulation of B-cell signaling pathways in the management of chronic GVHD.

There has been considerable clinical research into the treatment of GVHD, but to date the majority of the important trials testing the safety and efficacy of new drugs or a variety of other approaches have been conducted in countries other than Japan, in which new drugs and devices are more readily available. Jamil et al. [3] at the University of Alabama at Birmingham provide an up-to-date summary of the treatment of acute and chronic GVHD mainly in the United States and Europe. They also introduce some practical tips on the treatment of steroid-refractory GVHD based on their clinical experience.

The outcomes of allogeneic HSCT differ substantially between Japanese and Caucasian populations, because 
Japan shows a more frequent use of bone marrow in unrelated donor transplantation, a higher number of single-unit cord blood transplantations, a more frequent use of nonmyeloablative preconditioning for elderly patients, and a more homogeneous genetic background between donors and recipients. For these reasons, conclusions reached through studies of Caucasian populations do not necessarily apply to Japanese recipients. Murata [4] reviews studies on the prophylaxis and treatment of acute and chronic GVHD that have been conducted in Japan.

I believe that the articles in this "Progress in Hematology" provide readers with valuable information on the management of acute and chronic GVHD, which will hopefully lead to better outcomes for patients receiving allogeneic HSCT.

Acknowledgments This work was supported in part by a Health and Labour Sciences Research Grant (H25-Immunology-104 and
H26-Immunology-106) from the Ministry of Health, Labour and Welfare, Japan.

Conflict of interest The author declares no conflict of interest.

\section{References}

1. Shono Y, Docampo MD, Peled JU, Perobelli SM, Jenq RR. Intestinal microbiota-related effects on graft-versus-host disease. Int J Hematol. 2015. doi:10.1007/s12185-015-1781-5.

2. Nakasone H, Sahaf B, Miklos DB. Therapeutic benefits targeting B-cells in chronic graft-versus-host disease. Int J Hematol. 2015. doi:10.1007/s12185-015-1782-4.

3. Jamil MO, Mineishi S. State of the art acute and chronic GVHD treatment. Int J Hematol. 2015. doi:10.1007/s12185-015-1785-1.

4. Murata M. Prophylactic and therapeutic treatment of graftversus-host disease in Japan. Int J Hematol. 2015. doi:10.1007/ s12185-015-1784-2. 